Rapid Reviews COVID-19•

\title{
Review 1: "The Unequal Burden of the Covid-19 Pandemic: Racial/Ethnic Disparities in US Cause- Specific Mortality"
}

Ladan Golestaneh ${ }^{1}$

${ }^{1}$ Albert Einstein College of Medicine

Published on: Oct 21, 2021

License: Creative Commons Attribution 4.0 International License (CC-BY 4.0). 


\section{$\underline{\text { RR:C19 Evidence Scale rating by reviewer: }}$}

- Strong. The main study claims are very well-justified by the data and analytic methods used. There is little room for doubt that the study produced has very similar results and conclusions as compared with the hypothetical ideal study. The study's main claims should be considered conclusive and actionable without reservation.

\section{$* * * * * * * * * * * * * * * * * * * * * * * * * * * * * * * * * * * * * * *$}

Review: This manuscript, by Luck et al., links data from the National Center for Health Statistics to population estimates from the US census bureau to: 1)Measure the increase in all-cause mortality between 2019 and 2020 by age, race/ethnicity (limited to Black, White and Hispanic) and sex; and 2)Measure the attribution to COVID vs nonCOVID of any increase in death during this time period (as evidenced by the death certificate attestation). The authors further examine the distribution of increase in cause-specific mortality (the most common of which were diabetes, heart disease and other) stratified by age, race/ethnicity, and sex between the years. The premise of the study is that though mortality with COVID-19 disproportionately affected racial ethnic minorities as described in multiple reports, population level death rates had not been examined to describe the contribution of non-COVID, or misclassified COVID, deaths to these findings. Descriptive statistics are used to report on absolute differences in death events by cause between pre-COVID (2019) and COVID (2020) years, stratified by pre-selected demographic categories, to elucidate above. The authors then used ordinary least squares regression applied to trend in deaths between 2015-2019, to extrapolate expected death by race/ethnicity in 2020 for all cause and non-COVID mortality causes. The analytic methods are sound, and the study claims are justified. The authors successfully show that COVID-infection deaths disproportionately affected Hispanic men between ages 24 and 65, while deaths related to heart disease and diabetes were mostly responsible for the dramatic increase in mortality among Black individuals during the COVID period. They also found that though actual all-cause mortality rates were higher than predicted for 2020, actual and predicated mortality rates for heart disease and diabetes were within 1.6\% of each other (2019 and 2020) in all groups. The supported inferences made by this study are very relevant to the current understanding of the effects of COVID among racial/ethnic minorities. The strength of the study comes from its use of national data with full capture of all death events (in hospital and out) with minimal sampling bias which has been unavoidable in other reports of COVID outcomes concentrating on those afflicted by COVID only, or 
health-system/county or state level databases. The authors are clear in their descriptions of potential misclassification bias. Their explanations for their findings are supported by literature at large and by their data 